\title{
Cost-Effectiveness Analysis of Fosfomycin for Treatment of Uncomplicated Urinary Tract Infections in Ontario
}

\author{
Louise Perrault, ${ }^{1,2}$ Sybil Dahan, ${ }^{3}$ Ange Christelle Iliza, ${ }^{2,4}$ \\ Jacques LeLorier, ${ }^{2,4}$ and George G. Zhanel ${ }^{5}$ \\ ${ }^{1}$ International Market Access Consulting, Montreal, QC, Canada \\ ${ }^{2}$ University of Montréal, Faculty of Medicine, Montréal, QC, Canada \\ ${ }^{3}$ Triton Pharma Inc., Concord, ON, Canada \\ ${ }^{4}$ Centre de Recherche du Centre Hospitalier de l'Université de Montréal, Montreal, QC, Canada \\ ${ }^{5}$ Department of Medical Microbiology and Infectious Diseases, University of Manitoba, Winnipeg, MB, Canada
}

Correspondence should be addressed to Louise Perrault; louise.perrault@imarketaccess.com

Received 18 October 2016; Accepted 31 January 2017; Published 20 February 2017

Academic Editor: José A. Oteo

Copyright (C) 2017 Louise Perrault et al. This is an open access article distributed under the Creative Commons Attribution License, which permits unrestricted use, distribution, and reproduction in any medium, provided the original work is properly cited.

\begin{abstract}
Background and Objective. Bacterial resistance to antibiotics traditionally used to treat uncomplicated urinary tract infections (uUTIs) is rising in Canada. We compared the cost-per-patient in Ontario of including fosfomycin (an antibiotic with a low resistance profile) as an option for first-line empirical treatment of uUTIs with current cost of treatment with sulfonamides, fluoroquinolones, and nitrofurantoin. Methods. A decision-tree model was used to perform a cost-minimization analysis. All possible outcomes of a uUTI caused by bacterial species treated with either sulfonamides, fluoroquinolones, nitrofurantoin, or fosfomycin were included. Results. In the base case analysis, the cost-per-patient for treating uUTI with fosfomycin was $\$ 105.12$. This is similar to the cost-per-patient for each of the other currently reimbursed antibiotics (e.g., $\$ 96.19$ for sulfonamides, $\$ 98.85$ for fluoroquinolones, and $\$ 99.09$ for nitrofurantoins). The weighted average cost-per-patient for treating uUTI was not substantially elevated with the inclusion of fosfomycin in the treatment landscape ( $\$ 98.41$ versus $\$ 98.29$ with and without fosfomycin, resp.). The sensitivity analyses revealed that most $(88.34 \%)$ of the potential variation in cost was associated with the probability of progressing to pyelonephritis and hospitalization for pyelonephritis. Conclusion. Fosfomycin in addition to being a safe and effective agent to treat uUTI has a low resistance profile, offers a single-dose treatment administration, and is similar in cost to other reimbursed antibiotics.
\end{abstract}

\section{Introduction}

Urinary tract infections (UTIs) are common and, as such, are associated with substantial socioeconomic implications [1]. At least one-half of women report experiencing one or more UTIs in their lifetime [2,3]. Most UTIs are uncomplicated bacterial infections of the lower urinary tract (uUTIs) $[2,4]$. Escherichia coli is the uropathogen responsible for $75 \%$ or more of uUTIs [5-8]. Many patients with uUTIs experience considerable impact on their health-related quality of life (HRQoL), including impairments in functioning at work, school, and home $[9,10]$. Treatment of uUTIs is necessary not only to improve patient HRQoL, but also to reduce the risk of progression to pyelonephritis, a more serious condition [5].
Several antibiotics are used in Canada for treatment of uUTIs, including trimethoprim, trimethoprim-sulfamethoxazole (TMP-SMX), fluoroquinolones such as ciprofloxacin, nitrofurantoin, $\beta$-lactam antibiotics such as amoxicillinclavulanate (AMC), and fosfomycin, a phosphoric acid derivative that represents its own class of antibiotics [11-14].

In addition to an already high burden of illness, standard treatment options are being challenged by rising bacterial resistance rates. Common uropathogens, in particular display variable resistance patterns over time and by region [5]. This requires practitioners to be aware of current local resistance rates and to be vigilant in tracking regional changes before empirically prescribing antibiotics for the treatment of UTIs [5]. For decades, TMP-SMX was considered first-line therapy 
for uUTIs [3], but rising rates of TMP-SMX resistance among uropathogens, and consistent evidence that in vitro TMP-SMX resistance correlates with bacterial and clinical failure, have forced a reappraisal of its first-line use for uUTIs [5]. Today, the E. coli resistance rate to TMP-SMX in Canada exceeds the $20 \%$ threshold at which guidelines recommend against TMP-SMX use for uUTI treatment [5, 11, 15-17]. E. coli resistance to the commonly used fluoroquinolone ciprofloxacin was 19\% in Canada in 2007-2009 [16], approximately twice the threshold above which the Infectious Diseases Society of America/European Society for Microbiology and Infectious Diseases (IDSA/ESCMID) clinical practice guidelines no longer recommend use of fluoroquinolones for uUTIs [5]. Bacterial resistance has several detrimental consequences for patients and society, including treatment failure and risk of complications, increased length of stay in hospital facilities, increased expenses, and increased mortality [18].

As bacterial resistance rates to current first-line antibiotics continue to rise, there will be a need for options that are effective, safe, and not likely to further promote community resistance. Although there is limited experience with fosfomycin for treating uUTIs in Canada $[3,19]$, it has been used for decades in other countries [20]. A meta-analysis of seven randomized controlled trials involving 1272 female patients with uUTIs showed no difference in cure rate between fosfomycin and a variety of comparators (including TMPSMX, ciprofloxacin, and nitrofurantoin) [1]. Furthermore, the prevalence of $E$. coli resistance to fosfomycin is low (with an average resistance rate of $1.2 \%$ in a recent international survey) and has not shown a significant increase over time $[21,22]$.

To date, there is no published economic analysis that provides evidence on the relative cost-effectiveness of fosfomycin compared to recommended empirical therapy for uUTIs in Canada. The objective of this study was to examine the economic impact of using fosfomycin for first-line empirical treatment of uUTIs in adult women in Ontario, taking into account current antibiotic prices, treatment algorithms, event probabilities, and resistance rates.

\section{Methods}

2.1. Decision-Tree Model. Based on evidence of equivalent efficacy and safety in head-to-head trials [38-43] and a meta-analysis [1], we conducted a cost-minimization analysis (CMA) using a decision-tree model (TreeAge Pro 2015 software $^{\circledR}$, Williamstown, MA, USA). The model is based on a previous model of uUTI treatment caused by E. coli in women older than 18 years [27] and uses a payer perspective, specifically that of the Ontario Ministry of Health and Long-term Care. Only direct medical care costs are considered. The time horizon of the model included 2 treatment courses.

The model assumed that first-line empirical treatment of uUTIs would consist of one of the antibiotics currently reimbursed by the Ontario Drug Benefit Program (ODBP) [44] and recommended by recent guidelines [5, $13,14]$ for the treatment uUTIs: sulfonamides (TMP/SMX
DS $800 \mathrm{mg} / 160 \mathrm{mg}$, TMP $100 \mathrm{mg}$, or TMP $200 \mathrm{mg}$ ), fluoroquinolones (Cipro ${ }^{\circledR} \mathrm{XL}^{\mathrm{TM}} 500 \mathrm{mg}$, ciprofloxacin $250 \mathrm{mg}$ [generic], or norfloxacin $400 \mathrm{mg}$ ), or nitrofurantoins (MacroBID $^{\circledR} 100 \mathrm{mg}$, nitrofurantoin $50 \mathrm{mg}$ [generic], or nitrofurantoin $100 \mathrm{mg}$ [generic]).

The decision-tree model incorporates all possible outcomes of a uUTI caused by E. coli, Enterococcus species, Klebsiella pneumoniae, Proteus mirabilis, or all other bacterial species (combined) treated with either fluoroquinolones, sulfonamides, nitrofurantoin, or fosfomycin (Figure 1). For simplicity, the model does not include the probability of rare complications associated with specific antibiotics (e.g., Stevens-Johnson syndrome). The probability of vaginal yeast infections is also not included because this complication is generally treated with over-the-counter drugs that are not reimbursed by the ODBP.

The model compares all four treatments and considers the possibility that the bacterial species would be susceptible or resistant to each antibiotic. In the latter case, the UTI either spontaneously resolves or persists. Patients with persistent UTIs either progress to pyelonephritis (with outpatient or inpatient treatment) or, in the absence of pyelonephritis, are treated with a different antibiotic.

The model was internally validated. All codes and programming were validated by a third party who was not involved in creating the model.

2.2. Costs. Province-level data on drug costs were used in the model. For the base case scenario, the cost of treatment for each antibiotic used to treat uUTIs was computed based on prices in the Ontario Drug Benefit Formulary (ODBF) [44] and the recommended dosage for uUTIs, as outlined by recent clinical guidelines $[5,13,14]$. The cost of each comparator was obtained by calculating an average cost of treatment for the class of antibiotic weighted by the volume of claims reimbursed by the ODBP as reported by Pharmastat data (Table 1).

Data on healthcare resource use were obtained from multiple public sources including the Physician Services under the Health Insurance Act (April 1, 2016), Ontario Case Costing Initiative, and from Pharmastat provincial data $[24,45]$. Healthcare resource use varied by outcome. For uUTIs that resolved with first-line empirical treatment, a single clinic visit was included in the model. Urinalysis and urine cultures were not performed prior to first-line treatment. For uUTIs that persisted and required treatment with another antibiotic, the model included two clinic visits (one initial visit and one following failure of the first-line antibiotic) and a urine culture. Progression to pyelonephritis was assumed to occur during or after first-line treatment; thus, patients with pyelonephritis requiring outpatient treatment consumed the following resources: one initial clinic visit, one emergency room visit, two follow-up clinic visits (one 48 hours after initiation of treatment for pyelonephritis and one at the end of treatment), and one urine culture at the end of treatment to confirm resolution of infection. For patients with pyelonephritis requiring inpatient treatment, the model included an initial clinic visit, hospitalization for pyelonephritis, and a follow-up clinic visit with a urine 


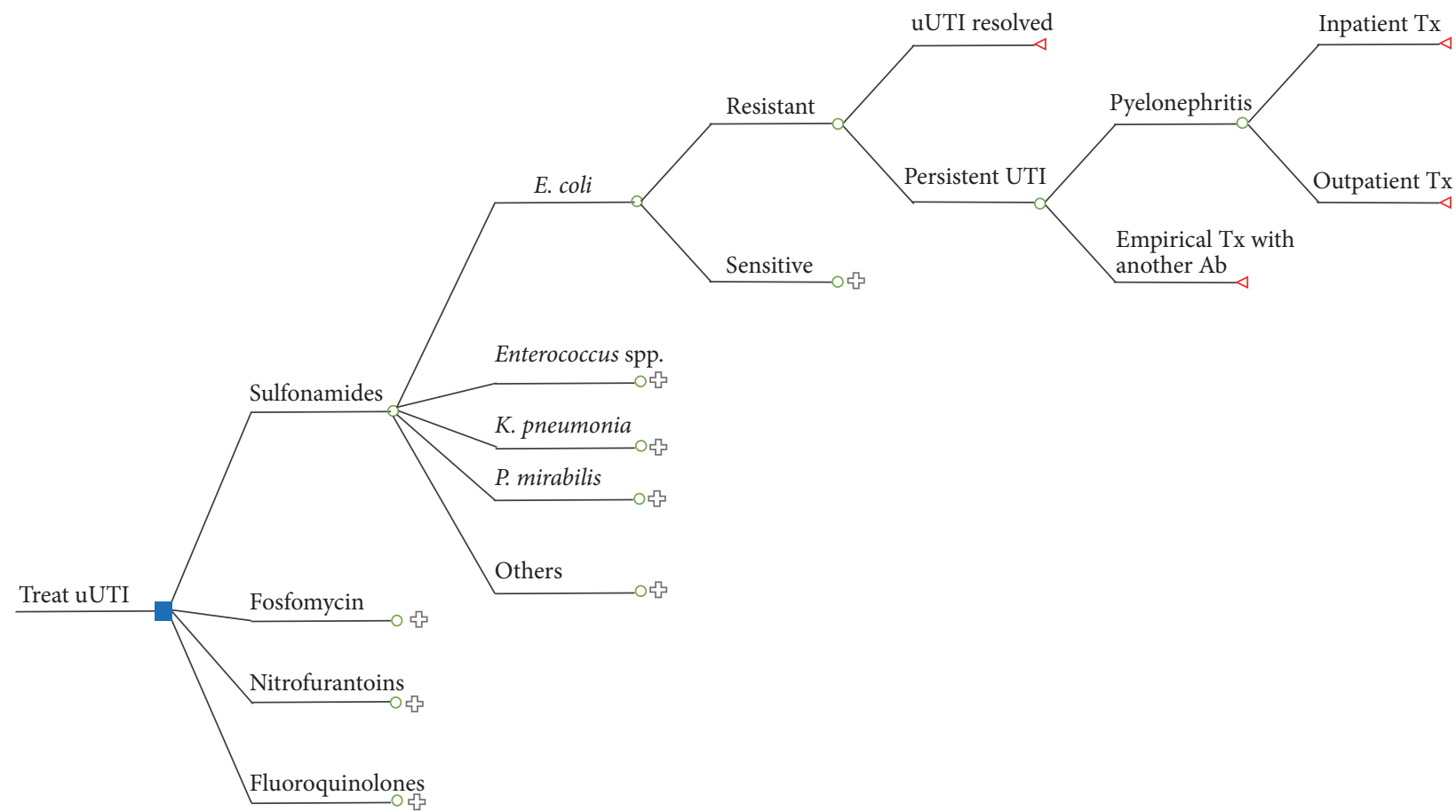

FIGURE 1: Decision-tree diagram showing intermediate and final outcomes for an uncomplicated E. coli UTI treated with a sulfonamide.

TABLE 1: Cost of antibiotics and healthcare resources used for the treatment of uUTIs and the ranges tested in the sensitivity analyses.

\begin{tabular}{lccc}
\hline Variable & Base case (CAD \$) & Range tested (CAD \$) & References \\
\hline Fosfomycin & 17.51 & $13.00-18.00$ & [23], Pharmastat \\
Sulfonamides & 0.95 & $0.88-2.79$ & [23], Pharmastat \\
Fluoroquinolones & 4.25 & $3.71-11.78$ & [23], Pharmastat \\
Nitrofurantoin & 10.54 & $4.68-11.12$ & [23], Pharmastat \\
Hospitalization for pyelonephritis & $6,918.15$ & $4,842.71-8,993.60$ & OCCI, hypothesis of plausible range ( $\pm 30 \%$ of base case) \\
Initial clinic visit & 77.20 & - & [24] \\
Follow-up clinic visit & 45.90 & - & Laboratoires Biron, oral communication, May 30 2016 \\
Urine culture & 45.00 & - & [24-26] \\
Emergency visit & $287.60^{\mathrm{a}}$ & - &
\end{tabular}

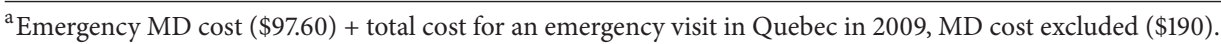

culture at the end of treatment to confirm resolution of infection.

First-line empirical treatment could be any one of the following: sulfonamide, fluoroquinolone, nitrofurantoin, or fosfomycin. Second-line treatment for persistent infections was either $\mathrm{Cipro}^{\circledR} \mathrm{XL}^{\mathrm{TM}} 500 \mathrm{mg}$ once per day for 3 days (if first treatment was not ciprofloxacin) or MacroBID ${ }^{\circledR}$ $100 \mathrm{mg}$ twice per day for 7 days (if first treatment was ciprofloxacin). Pyelonephritis was treated with either Cipro ${ }^{\circledR}$ $\mathrm{XL}^{\mathrm{TM}} 500 \mathrm{mg} / \mathrm{g}$ once per day for 7 days (if first treatment was not ciprofloxacin) or Clavulin ${ }^{\circledR} 500 / 125 \mathrm{~g}$ three times per day for 14 days (if first treatment was ciprofloxacin).

Costs associated with productivity loss are not included in the analysis; only direct medical care costs are considered.

2.3. Probabilities. Probabilities were obtained from published estimates. Probabilities of resistance for fluoroquinolones, sulfonamides, and nitrofurantoin were obtained from the
CANWARD surveillance study 2007-2011 [15] and the CANWARD surveillance study 2007-2009 (Table 2) [16]. Resistance rates for fosfomycin were obtained from the ARESC (Antimicrobial Resistance Epidemiological Survey on Cystitis) study [22]. Probabilities for effectiveness were assumed to be equal for all antibiotics based on the meta-analysis published by Falagas et al. (2010) [1]. Effectiveness was assumed to be reduced by $20 \%$ for resistant isolates [33]. Probabilities of pyelonephritis (i.e., pyelonephritis due to initial treatment failure and hospitalization for pyelonephritis) were obtained from different sources [27, 28, 34-36].

2.4. Sensitivity Analyses. Variables suspected to have the most influence on the expected value of cost-per-patient were subjected to a deterministic one-way sensitivity analysis (DSA). Where possible, ranges for variables were extracted from the literature or other public sources. In the absence of a reliable source, a hypothesis of the plausible range was used. 
TABLE 2: Probability values used in the model for decision-tree nodes and ranges used in the sensitivity analyses.

\begin{tabular}{|c|c|c|c|}
\hline Variable & Base case & Range tested & References \\
\hline Sensitive isolate: UTI resolution (clinical cure) & 0.94 & - & {$[27-32]$} \\
\hline Resistant isolate: UTI resolution (clinical cure) & 0.74 & - & [33] \\
\hline Pyelonephritis due to initial treatment failure & 0.04 & $0-0.08$ & $\begin{array}{l}{[27,28,34,35]} \\
\text { hypothesis of plausible } \\
\text { range }\end{array}$ \\
\hline Hospitalization for pyelonephritis & 0.20 & $0-0.40$ & $\begin{array}{l}\text { [36], hypothesis of } \\
\text { plausible range }\end{array}$ \\
\hline $\begin{array}{l}\text { Empirical extended treatment due to initial } \\
\text { treatment failure (switch from initial treatment to } \\
\text { another antibiotic) }\end{array}$ & $\begin{array}{l}0.75 / 0.25 \\
(0.25 / 0.75 \text { for } \\
\text { ciprofloxacin })\end{array}$ & - & {$[27,28,35]$} \\
\hline \multicolumn{4}{|l|}{ Pathogens present in UTI } \\
\hline E. coli & 0.841 & $0.767-0.918$ & {$[7,22,37]$} \\
\hline Enterococcus spp. & 0.028 & - & {$[37]$} \\
\hline K. pneumoniae & 0.038 & - & {$[37]$} \\
\hline P. mirabilis & 0.026 & - & [37] \\
\hline Treatment with fosfomycin & 0.0177 & $0-0.250$ & $\begin{array}{c}\text { Pharmastat, hypothesis } \\
\text { of plausible range }\end{array}$ \\
\hline Treatment with sulfonamides & 0.2566 & - & Pharmastat \\
\hline Treatment with nitrofurantoins & 0.5410 & - & Pharmastat \\
\hline Treatment with fluoroquinolones & 0.1848 & - & Pharmastat \\
\hline E. coli resistance to fosfomycin & 0.006 & $0.004-0.012$ & {$[6,21,22]$} \\
\hline E. coli resistance to sulfonamides (TMP-SMX) & $0.267^{\mathrm{a}}$ & $0.141-0.294$ & {$[15,16]$} \\
\hline $\begin{array}{l}\text { E. coli resistance to fluoroquinolones } \\
\text { (ciprofloxacin) }\end{array}$ & $0.218^{\mathrm{a}}$ & $0.191-0.245$ & {$[6,15,22]$} \\
\hline E. coli resistance to nitrofurantoin & $0.011^{\mathrm{a}}$ & $0.0016-0.019$ & {$[15,16,22]$} \\
\hline
\end{tabular}

TMP-SMX, trimethoprim-sulfamethoxazole; UTI, urinary tract infection.

${ }^{a}$ Derived from urinary, respiratory, wound, and blood isolates.

The base case values and the ranges tested in the sensitivity analyses are shown in Tables 1 and 2.

\section{Interpretation}

This study uses a cost-minimization analysis (CMA) to identify the most affordable options for the treatment of uUTI in Ontario, taking into account both cost-per-dose and costs associated with antibiotic resistance.

3.1. Analysis and Results. With the current cost of fosfomycin of \$17.51, the cost-per-patient for treating uUTIs with this drug was determined to be $\$ 105.12$. This is comparable to the cost-per-patient for each of the other currently reimbursed antibiotics (Figure 2).

The weighted average cost-per-patient for treating uUTI in Ontario was also estimated based on 2 scenarios: with and without fosfomycin. In this analysis, cost-per-patient was obtained by replacing the decision node of the tree with a chance node which identifies probabilities of events. The probabilities were the market share of each alternative treatment. Thus, the weighted average cost takes into account the antibiotics currently reimbursed by the ODB and their respective market shares. The weighted average cost-per-patient for treating uUTI in Ontario is \$98.29 (Figure 3). Market shares are based on Ontario Pharmastat data.

Incorporating fosfomycin into the mix of treatments reimbursed in Ontario results in a weighted average cost-perpatient of $\$ 98.41$ (Figure 4). For this analysis, the estimated market share of fosfomycin was $1.77 \%$ as reported by Pharmastat. The impact of reimbursing fosfomycin in the Ontario treatment landscape has a negligible impact on the weighted cost-per-patient and provides physicians with an effective alternate treatment option.

3.2. Results of Uncertainty Analysis. Sensitivity analyses were performed based on the decision and chance nodes of the decision tree. The tornado diagram revealed that the probability of developing pyelonephritis has the largest potential effect on the expected value (cost-per-patient) of the model (Figure 5). Other variables that had a potential effect on costper-patient were as follows:

(i) Probability of hospitalization for pyelonephritis

(ii) Probability of resistance of E. coli to TMP-SMX

(iii) Cost of hospitalization for pyelonephritis 


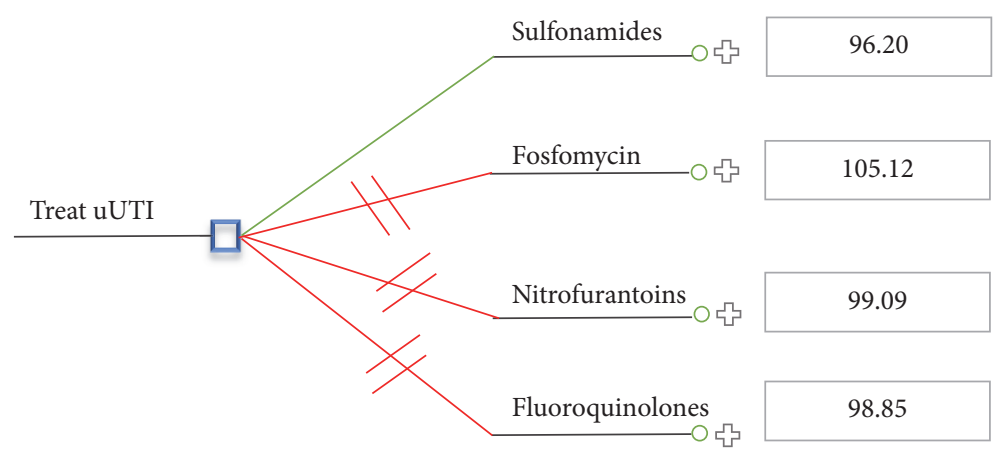

Sulfonamides: 96.20

FIGURE 2: Cost-per-patient for treating uUTI in Ontario.

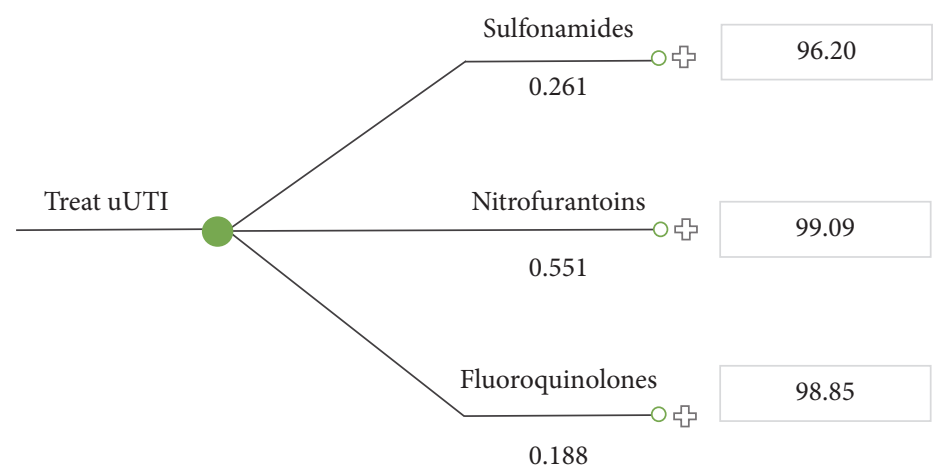

Treat uUTI: 98.29

FIGURE 3: Weighted average cost-per-patient for treating uUTI in Ontario without fosfomycin.

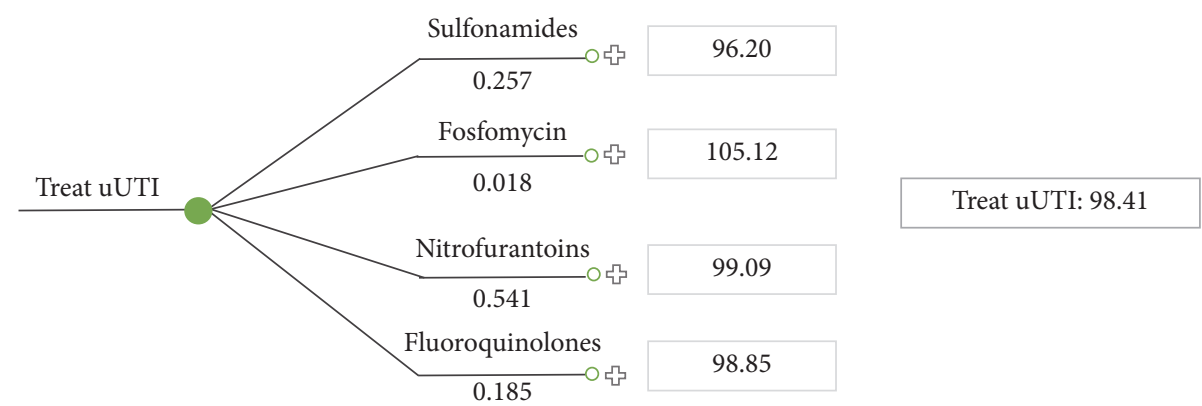

FIGURE 4: Weighted average cost-per-patient for treating uUTI in Ontario with fosfomycin.

Probability of pyelonephritis (\%)

Probability of hospitalization for pyelonephritis (\%)

Probability of resistance of E. coli to TMP-SMX (\%)

Hospitalization for pyelonephritis (\$)

Nitrofurantoins cost (\$)

Sulfonamides (\$)

Probability of E. coli infection (\%)

Probability of resistance of $E$. coli to nitrofurantoins (\%)

Probability of resistance of E. coli to fosfomycin (\%)

Fluoroquinolones cost (\$)

Fosfomycin cost (\$)

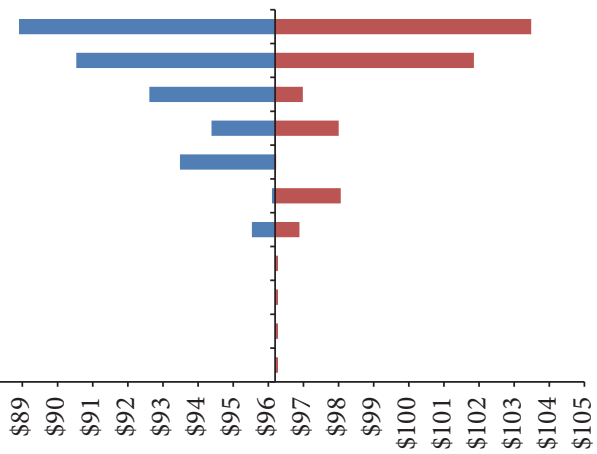

FIGURE 5: Tornado diagram comparing the results of the one-way sensitivity analyses of variables potentially affecting the expected value of the cost-per-patient (decision node). 


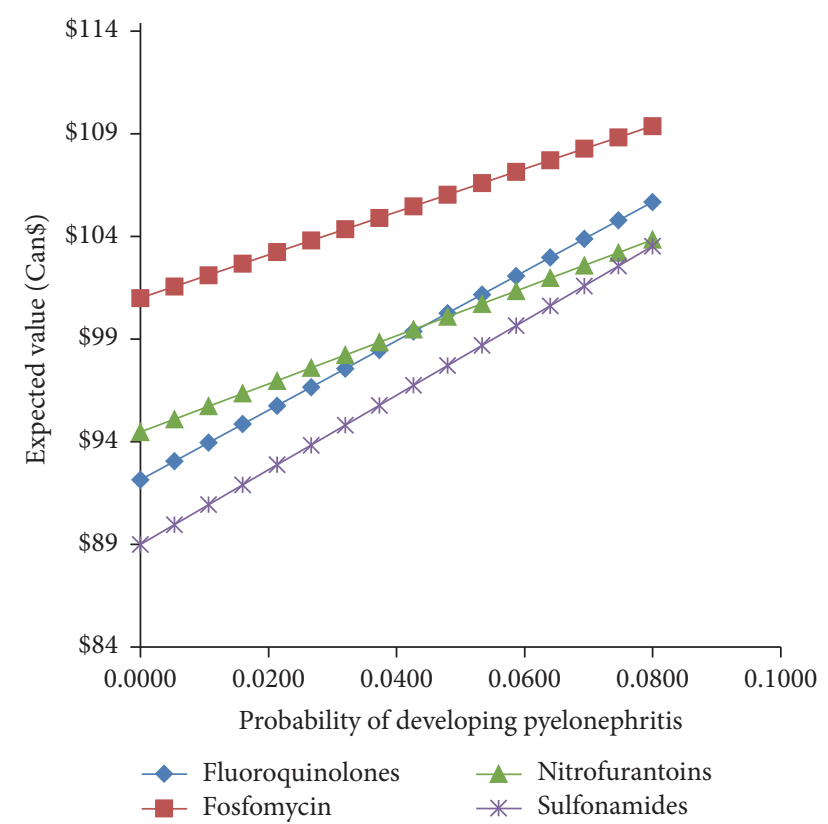

Figure 6: Comparison of the expected value of the cost-perpatient when treating uUTI with fluoroquinolones, sulfonamides, nitrofurantoin, and fosfomycin versus the probability of developing pyelonephritis.

\section{(iv) Cost of nitrofurantoin}

(v) Probability of being infected by E. coli

Variables accounting for $93.18 \%$ of the potential variation in cost-per-patient were the probability of progressing to pyelonephritis (55.13\%), having to be hospitalized for pyelonephritis (33.17\%), and contracting an infection with TMP-SMX-resistant E. coli (4.87\%). Detailed results of the one-way sensitivity analyses are presented in Appendix.

Figure 6 compares the cost-per-patient for treating uUTI with a fluoroquinolone, sulfonamide, nitrofurantoin, or fosfomycin with various probabilities of progression to pyelonephritis. At all levels of risk of progression to pyelonephritis, fosfomycin is associated with the highest cost-per-patient. As the risk of pyelonephritis increases, the expected values of other antimicrobial treatments approach that of fosfomycin. Note that the base case value for progression to pyelonephritis is $4 \%[27,28,34,35]$.

Figure 7 compares the cost-per-patient for treating uUTI with one of the 4 antimicrobial treatments with the probability of hospitalization due to pyelonephritis. At all probabilities, fosfomycin is associated with the highest cost. Note that the base case value for the probability of hospitalization due to pyelonephritis is $20 \%$ [36].

Figure 8 compares the expected cost-per-patient for the treatment of a uUTI with a fluoroquinolone, sulfonamide, nitrofurantoin, or fosfomycin with the prevalence of TMPSMX-resistant E. coli. The cost-per-patient when using a sulfonamide steadily increases with increasing resistance probabilities. The cost-per-patient of the other antibiotics is not affected by this variable. Note that the base case value for E. coli resistance to sulfonamides is $26.7 \%$ [15].

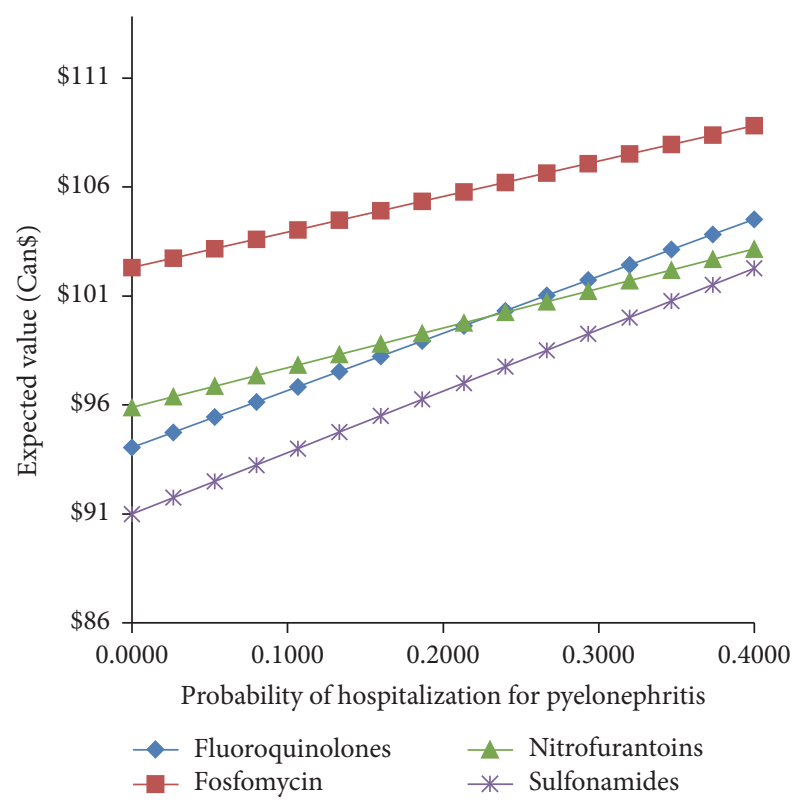

Figure 7: Comparison of the expected value of the cost-per-patient when treating uUTI with fluoroquinolones, sulfonamides, nitrofurantoin, and fosfomycin versus the probability of hospitalization for pyelonephritis.

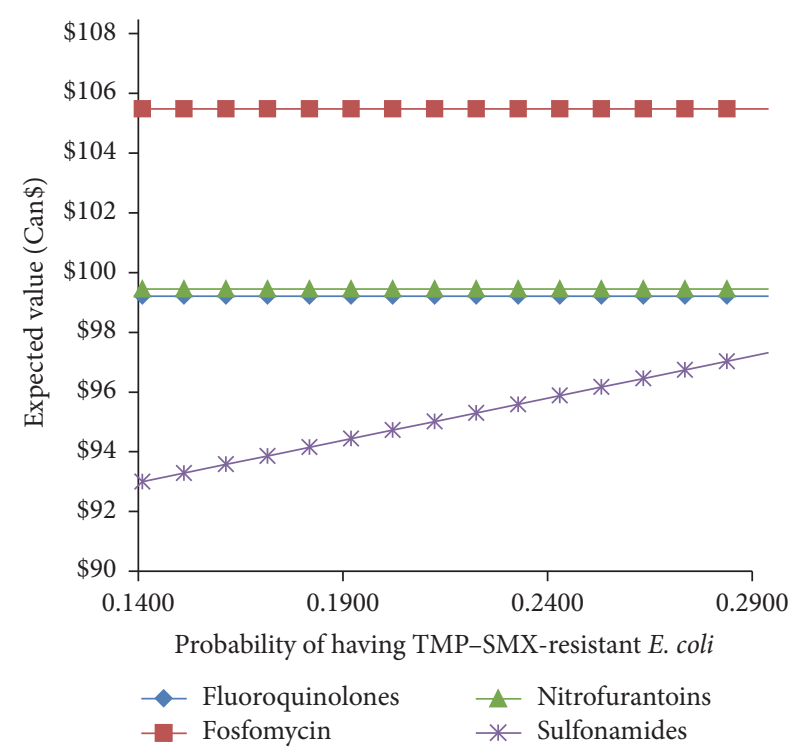

FIgURE 8: Comparison of the expected value of the cost-perpatient when treating uUTI with fluoroquinolones, sulfonamides, nitrofurantoin, and fosfomycin versus the probability of a uUTI with TMP-SMX-resistant E. coli.

A tornado diagram was also developed using the oneway sensitivity analyses of the chance node instead of the decision node of the decision tree (Figure 9). The major difference in the results of this sensitivity analysis was that the cost of nitrofurantoins was the third highest contributor to the potential variation in cost-per-patient-meaning that the cost of nitrofurantoins has a great influence on the cost of treating patients with uUTI covered by the ODB. Recall that, 


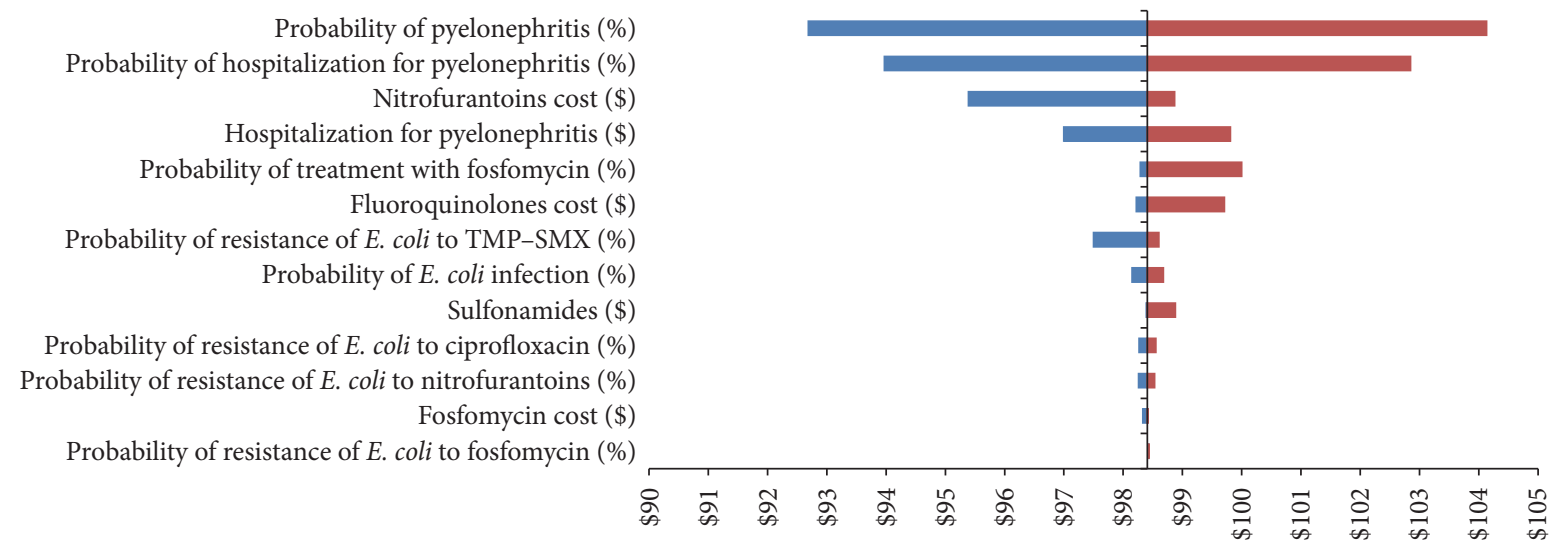

FIGURE 9: Tornado diagram comparing the results of the one-way sensitivity analyses of variables potentially affecting the expected value of cost-per-patient (chance node).

in the decision node analysis, the third highest contributor was probability of contracting an infection with TMP-SMXresistant E. coli.

\section{Discussion}

Although there is limited experience with fosfomycin for treating uUTI in Canada [3], it has been used successfully for decades in other countries $[19,20]$. Fosfomycin is a phosphoric acid derivative that represents its own class of antibiotics; no other member of this class is currently approved by regulatory agencies worldwide $[19,20]$.

Resistance rates for ciprofloxacin and TMP-SMX (INESSS first-line recommendations for treatment of uUTI) were much lower a decade ago than they are now. The ECO-SENS survey, conducted in 1999-2000, showed E. coli resistance rates of $0 \%$ for ciprofloxacin and $12 \%$ for TMP-SMX in Canada ( $n=166$, urinary isolates) [6]. As of 2009, the CANWARD surveillance initiative revealed that E. coli resistance to TMP-SMX in urinary isolates was $24.3 \%$ and had undergone a significant increase over just a 3-year period-the resistance rate was $18.6 \%$ in 2007 (data derived from urine samples of 2943 outpatients and inpatients, $P=0.02$ ) [16]. Recent data from the CANWARD surveillance initiative (2011) focusing on resistance rates regardless of source (i.e., not specific to uropathogens) revealed that $E$. coli resistance to ciprofloxacin and TMPSMX is quite high in Canada (26.9\% for ciprofloxacin and $29.3 \%$ for TMP-SMX, $N=646$ ). Ontario resistance rates in 2011 were similar to those seen across Canada $(24.6 \%$ for ciprofloxacin and $28.1 \%$ for TMP-SMX, $N=167$ ) [46]. It is clear that antibiotic resistance is on the rise, particularly for ciprofloxacin and TMP-SMX.

In contrast, the ECO-SENS survey (conducted in 19992000 and then again in 2007-2008) showed that the initial low resistance rate of $E$. coli to fosfomycin did not increase significantly over time. In E. coli isolates from 5 European countries, the resistance rate to fosfomycin was $0.4 \%$ in $1999-2000$ and $1.2 \%$ in 2007-2008 [21]. This consistently low resistance rate may be due to the fact that fosfomycin resistance comes at a biological cost to microorganisms [19].
Furthermore, the low resistance of E. coli in the ECO-SENS survey could be explained by the exclusion of hospitalized patients during the two weeks before the onset of symptoms and data from the CANWARD surveillance initiative were based on samples from outpatients and inpatients. Microbiological studies have shown that fosfomycin-resistant strains of E. coli are "biologically impaired"-they take up to $39 \%$ longer to replicate and they have a $38 \%$ reduction in the ability to adhere to uroepithelial cells. These resistant strains are unable to compete with susceptible strains in the environment, possibly contributing to the low rates of fosfomycin resistance observed over time [19, 47].

Fosfomycin also has a low propensity for cross-resistance, which is likely due to the fact that it differs from other antibiotics in its general chemical structure and site of action $[19,48]$. The unique mechanism of action of fosfomycin appears to underlie its low rate of cross-resistance. This mechanism of action, which inhibits the early stages of cell wall synthesis [49], reduces the risk of cross-resistance with other antibacterial agents, making fosfomycin a good choice for the treatment of multidrug-resistant strains and leaving options available for further therapies [19, 47]. In the ECO-SENS survey, cross-resistance in E. coli was observed with ampicillin and SMX, alone or linked with resistance to TMP and TMP-SMX. A multiple-resistant phenotype involving fluoroquinolone resistance was also noted. The ECO-SENS authors concluded that resistance to any agent was associated with increased resistance to all other agents tested, fosfomycin being the only exception [50].

In addition to the biological aspect of the low fosfomycin resistance rate, single-dose antibiotics are associated with a high degree of compliance and greater convenience, which may help prevent resistance from developing [19, 51, 52]. Maintenance of $100 \%$ adherence to optimized dosing regimens is critical for ensuring antimicrobial efficacy of the drug and prevention of resistance. Experts have recommended that underdosing be prevented to "suppress or decrease the potential amplification of resistant mutant subpopulations" and that regimens should be as short as possible in order to maximally suppress resistance [52]. Other potential benefits 
of single-dose therapy include directly observed therapy (i.e., compliance is not an issue) and fewer side effects [53].

Since resistance patterns of $E$. coli strains causing uUTI vary considerably across regions and can be well beyond the resistance threshold for certain antibiotics, the IDSA/ESCMID guidelines note that a specific treatment recommendation may not be universally suitable $[5,46]$. The guidelines now include fosfomycin as one of the recommended first-line options for the treatment of uUTIs along with nitrofurantoin, TMP-SMX, and pivmecillinam [5]. In May 12, 2016, the FDA made recommendations resulting from a November 5, 2015, meeting, (Joint Meeting of the Antimicrobial Drugs Advisory Committee [formerly known as the Anti-Infective Drugs Advisory Committee] and Drug Safety and Risk Management Advisory Committee Meeting) that, based on risk versus benefit, fluoroquinolones (such as ciprofloxacin and levofloxacin) should not be used first line for the treatment of uUTI; rather nitrofurantoin, TMP-SMX, and fosfomycin should be used instead [54].

In this new world of rapidly advancing microbial resistance, an additional antibiotic in the armamentarium, especially one with a low resistance profile, may be of substantial benefit to Ontario practitioners and patients.

4.1. Summary of Results. This analysis revealed that the costper-patient for treating uUTI in Ontario is similar for all 4 antibiotics, ranging from $\$ 96.19$ for sulfonamides to $\$ 105.12$ for fosfomycin. The average cost-per-patient for treating uUTI in Ontario with all 4 reimbursed antimicrobial agents (fosfomycin, fluoroquinolones, sulfonamides, or nitrofurantoin), weighted by market share, is $\$ 98.41$ (versus $\$ 98.29$ without fosfomycin).

Most of the potential variability in cost-per-patient (93.18\%) is associated with the probability of progressing to pyelonephritis $(55.13 \%)$, having to be hospitalized for pyelonephritis $(33.17 \%)$, and contracting an infection with TMP-SMX-resistant E. coli (4.87\%).

4.2. Study Limitations. There were limitations to this study. First, efficacy was assumed to be equal based on the metaanalysis conducted by Falagas et al. (2010) [1]. However, this analysis was only focused on comparing fosfomycin with other antibiotics. Meta-analyses providing efficacy comparisons between the other antimicrobial agents were not identified in the literature. Similarly, we were unable to locate sources for the efficacy of each agent in susceptible versus resistant infections.

Secondly, the only published data we had for the probability of developing pyelonephritis was from a US source. As the model was the most sensitive to this variable, we cannot definitively determine its reliability in Canada.

4.3. Generalizability. This model has been constructed to specifically address the empirical treatment of uUTI in women older than 18 years of age. This population is aligned with the current approved indication of fosfomycin in Canada.

The data used to populate the model were derived from Ontario and/or Canadian sources wherever possible. Costs and market share of currently reimbursed antibiotics were derived from provincial data, providing an accurate view of the present market.

Consultation with experts on clinical practice patterns and costs provided an additional layer of real-world validity.

4.4. Health Services Impact. Since the availability of fosfomycin does not significantly increase the weighted average cost of treatment per patient, reimbursement of this antibiotic in Ontario has a negligible effect on the provincial healthcare budget. The societal implications are, however, substantial. Providing access to an additional antibiotic, especially one with a low resistance profile like fosfomycin, may benefit both Ontario practitioners and their patients. With rising rates of resistance to fluoroquinolones and sulfonamides, these "staple" antibiotics are no longer broadly considered appropriate first-line empirical therapies by international experts [5]. This shift of recommended treatments in the armamentarium leaves physicians in Ontario with fewer options for treating uUTIs. Reimbursement of fosfomycin by the ODBP would allow physicians to effectively treat patients with uUTIs while ensuring that community resistance to important antibiotics (fluoroquinolones and sulfonamides) is not further increased in Ontario.

4.5. Future Research. Further research into the efficacy of fluoroquinolones, sulfonamides, and nitrofurantoin in resistant infections would be beneficial for future pharmacoeconomic evaluations, especially since the rates of resistance for some of these agents have risen drastically in a short period of time. Additionally, head-to-head studies of antibiotic efficacy and safety in current uUTI patient populations would provide much needed data for developing accurate probabilities of treatment success/failure.

\section{Conclusions}

Fosfomycin in addition to being a safe and effective agent to treat uUTI has a low resistance profile, offers a singledose treatment regimen, and is comparable in cost to other reimbursed antibiotics.

\section{Appendix}

See Table 3.

\section{Competing Interests}

Louise Perrault, Sybil Dahan, George G. Zhanel, and Jacques LeLorier have served as consultants to Triton Pharma and received funding from Triton Pharma for their contributions to this analysis.

\section{Authors' Contributions}

Louise Perrault and Sybil Dahan conceived of the economic model, developed it, and produced the analyses. George G. Zhanel and Jacques LeLorier provided microbiology and 
TABLE 3: Results of tornado diagram (decision node).

\begin{tabular}{|c|c|c|c|c|c|c|c|c|}
\hline Variable & Range & $\begin{array}{c}\text { Low } \\
\text { EV }\end{array}$ & $\begin{array}{l}\text { High } \\
\text { EV }\end{array}$ & Spread & Spread $^{2}$ & $\begin{array}{c}\text { Risk } \\
(\%)\end{array}$ & $\begin{array}{c}\text { Cumulative } \\
\text { risk (\%) }\end{array}$ & $\begin{array}{c}\text { Variable } \\
\text { index }\end{array}$ \\
\hline $\begin{array}{l}\text { Probability of } \\
\text { developing PN }\end{array}$ & 0.0 to 0.08 & 88.93 & 103.46 & 14.530 & 211.121 & 0.553 & 0.553 & 0 \\
\hline $\begin{array}{l}\text { Probability of } \\
\text { hospitalization } \\
\text { for PN }\end{array}$ & 0.0 to 0.4 & 90.56 & 101.83 & 11.270 & 127.013 & 0.332 & 0.885 & 1 \\
\hline $\begin{array}{l}\text { Probability of } \\
\text { resistance of } E \text {. } \\
\text { coli to } \\
\text { TMP-SMX }\end{array}$ & 0.141 to 0.294 & 92.64 & 96.96 & 4.320 & 18.662 & 0.049 & 0.934 & 2 \\
\hline $\begin{array}{l}\text { Cost of } \\
\text { hospitalization } \\
\text { for PN }\end{array}$ & $\begin{array}{c}4842.71 \text { to } \\
8993.60\end{array}$ & 94.41 & 97.98 & 3.570 & 12.745 & 0.033 & 0.967 & 3 \\
\hline $\begin{array}{l}\text { Cost of } \\
\text { nitrofurantoin }\end{array}$ & 4.68 to 11.12 & 93.51 & 96.20 & 2.690 & 7.236 & 0.019 & 0.986 & 4 \\
\hline $\begin{array}{l}\text { Probability of } \\
\text { being infected } \\
\text { by } E \text {. coli }\end{array}$ & 0.767 to 0.918 & 95.56 & 96.86 & 1.300 & 1.690 & 0.004 & 1 & 5 \\
\hline $\begin{array}{l}\text { Probability of } \\
\text { resistance of } E \text {. } \\
\text { coli to } \\
\text { nitrofurantoin }\end{array}$ & 0.0016 to 0.019 & 96.20 & 96.20 & 0.000 & 0.000 & 0.000 & 1 & 6 \\
\hline $\begin{array}{l}\text { Probability of } \\
\text { resistance of } E \text {. } \\
\text { coli to } \\
\text { fosfomycin }\end{array}$ & 0.004 to 0.012 & 96.20 & 96.20 & 0.000 & 0.000 & 0.000 & 1 & 7 \\
\hline $\begin{array}{l}\text { Probability of } \\
\text { resistance of } E \text {. } \\
\text { coli to } \\
\text { ciprofloxacin }\end{array}$ & 0.191 to 0.245 & 96.20 & 58.69 & 0 & 0 & 0.000 & 1 & 8 \\
\hline $\begin{array}{l}\text { Cost of } \\
\text { ciprofloxacin }\end{array}$ & 3.71 to 11.78 & 96.20 & 96.20 & 0.000 & 0.000 & 0.000 & 1 & 9 \\
\hline $\begin{array}{l}\text { Cost of } \\
\text { fosfomycin }\end{array}$ & 13.0 to 18.0 & 96.20 & 58.69 & 0 & 0 & 0.000 & 1 & 10 \\
\hline
\end{tabular}

$\mathrm{EV}$, expected value; PN, pyelonephritis.

clinical expertise. All authors contributed to interpretation of the data, helped to draft the report, and read and approved the final version.

\section{Acknowledgments}

Medical writing and editorial support were provided by Sabrina Smith, Church Point, NS, Canada, funded by International Market Access Consulting and Triton Pharma (acquired by Paladin Labs Inc. in January 2014).

\section{References}

[1] M. E. Falagas, E. K. Vouloumanou, A. G. Togias et al., "Fosfomycin versus other antibiotics for the treatment of cystitis: a meta-analysis of randomized controlled trials," Journal of Antimicrobial Chemotherapy, vol. 65, no. 9, pp. 1862-1877, 2010.

[2] R. Colgan and M. Williams, "Diagnosis and treatment of acute uncomplicated cystitis," American Family Physician, vol. 84, no. 7, pp. 771-776, 2011.
[3] L. Nicolle, P. A. M. Anderson, J. Conly et al., "Uncomplicated urinary tract infection in women: current practice and the effect of antibiotic resistance on empiric treatment," Canadian Family Physician, vol. 52, pp. 612-618, 2006.

[4] T. M. Hooton, "Pathogenesis of urinary tract infections: an update," Journal of Antimicrobial Chemotherapy, vol. 46, S1, pp. $1-7,2000$.

[5] K. Gupta, T. M. Hooton, K. G. Naber et al., "International clinical practice guidelines for the treatment of acute uncomplicated cystitis and pyelonephritis in women: a 2010 update by the Infectious Diseases Society of America and the European Society for Microbiology and Infectious Diseases," Clinical Infectious Diseases, vol. 52, no. 5, pp. e103-e120, 2011.

[6] G. Kahlmeter, "An international survey of the antimicrobial susceptibility of pathogens from uncomplicated urinary tract infections: the ECO.SENS project," Journal of Antimicrobial Chemotherapy, vol. 51, no. 1, pp. 69-76, 2003.

[7] T. Mazzulli, "Antimicrobial resistance trends in common urinary pathogens," The Canadian Journal of Urology, vol. 8, pp. $2-5,2001$. 
[8] K. G. Naber, G. Schito, H. Botto, J. Palou, and T. Mazzei, "Surveillance study in Europe and Brazil on clinical aspects and antimicrobial resistance epidemiology in females with cystitis (ARESC): implications for empiric therapy," European Urology, vol. 54, no. 5, pp. 1164-1178, 2008.

[9] S. L. Bermingham and J. F. Ashe, "Systematic review of the impact of urinary tract infections on health-related quality of life," BJU International, vol. 110, no. 11, pp. E830-E836, 2012.

[10] J. C. Nickel, J. C. Lee, J. E. Grantmyre, and D. Polygenis, "Natural history of urinary tract infection in a primary care environment in Canada," The Canadian Journal of Urology, vol. 12, no. 4, pp. 2728-2737, 2005.

[11] Institut National D'excellence En Santé Et En Services Sociaux (Inesss), "Guides cliniques en antibiothérapie-Série I: Infections urinaires chez l'adulte," 2009, http://www.inesss.qc.ca/ fileadmin/doc/CDM/UsageOptimal/Guides-serieI/CdM-Antibiol-InfectionsUrinaires-Adultes-fr.pdf.

[12] Paladin Labs Inc, Product Monograph: Monurol ${ }^{\circledR}$ (Fosfomycin Tromethamine) $3 g$ Sachet, Paladin Labs Inc, St. Laurent, Canada, 2015.

[13] Anti-Infective Review Panel, Anti-Infective Guidelines for Community-Acquired Infections, 2013.

[14] E. Blondel-Hill and S. Fryters, Bugs \& Drugs: an Antimicrobial/Infectious Diseases Reference, Alberta Health Services, Alberta, Canada, 2012.

[15] G. G. Zhanel, H. J. Adam, M. R. Baxter et al., "Antimicrobial susceptibility of 22746 pathogens from Canadian hospitals: results of the CANWARD 2007-11 study," The Journal of antimicrobial chemotherapy, vol. 68, pp. i7-i22, 2013.

[16] J. A. Karlowsky, P. R. S. Lagacé-Wiens, P. J. Simner et al., "Antimicrobial resistance in urinary tract pathogens in Canada from 2007 to 2009: CANWARD surveillance study," Antimicrobial Agents and Chemotherapy, vol. 55, no. 7, pp. 3169-3175, 2011.

[17] W. J. McIsaac, T. Mazzulli, J. Permaul, R. Moineddin, and D. E. Low, "Community-acquired antibiotic resistance in urinary isolates from adult women in Canada," Canadian Journal of Infectious Diseases and Medical Microbiology, vol. 17, no. 6, pp. 337-340, 2006.

[18] Institut National D’Excellence En Santé Et En Services Sociaux (Inesss), Guides Cliniques en Antibiothérapie-Série II: Information Sheet on Bacterial Resistance, 2012, http://www .inesss.qc.ca/fileadmin/doc/INESSS/Outils/Guides_antibio_II/ GUIDE_RESISTANCE_2012_web_EN.pdf.

[19] G. G. Zhanel, A. J. Walkty, and J. A. Karlowsky, "Fosfomycin: a first-line oral therapy for acute uncomplicated cystitis," Canadian Journal of Infectious Diseases and Medical Microbiology, vol. 2016, Article ID 2082693, 10 pages, 2016.

[20] M. Popovic, D. Steinort, S. Pillai, and C. Joukhadar, "Fosfomycin: an old, new friend?" European Journal of Clinical Microbiology and Infectious Diseases, vol. 29, no. 2, pp. 127-142, 2010.

[21] G. Kahlmeter and H. O. Poulsen, "Antimicrobial susceptibility of Escherichia coli from community-acquired urinary tract infections in Europe: the ECO-SENS study revisited," International Journal of Antimicrobial Agents, vol. 39, no. 1, pp. 45-51, 2012.

[22] G. C. Schito, K. G. Naber, H. Botto et al., "The ARESC study: an international survey on the antimicrobial resistance of pathogens involved in uncomplicated urinary tract infections," International Journal of Antimicrobial Agents, vol. 34, no. 5, pp. 407-413, 2009.
[23] Ministry of Health and Long-Term Care, Ontario Drug Benefit Formulary/Comparative Drug Index, 2016, https://www .formulary.health.gov.on.ca/formulary/.

[24] Ministry of Health and Long-Term Care, Schedule of Benefits: Physician Services under the Health Insurance Act, 2016.

[25] Régie De Lassurance Maladie Du Québec (Ramq), "Manuel des médecins omnipraticiens," Tech. Rep. 100, RAMQ, Quebec, Canada, 2015.

[26] Ordre Des Infirmières Et Infirmiers Auxiliaires Du Québec (OIIAQ), Les Services d'Urgence au Québec: Caractéristiques et Utilisation des Infirmières Auxiliaires, OIIAQ, Quebec, Canada, 2009.

[27] J. A. Mckinnell, N. S. Stollenwerk, C. W. Jung, and L. G. Miller, "Nitrofurantoin compares favorably to recommended agents as empirical treatment of uncomplicated urinary tract infections in a decision and cost analysis," Mayo Clinic Proceedings, vol. 86, no. 6, pp. 480-488, 2011.

[28] H. C. Barry, M. H. Ebell, and J. Hickner, "Evaluation of suspected urinary tract infection in ambulatory women: a costutility analysis of office-based strategies," Journal of Family Practice, vol. 44, no. 1, pp. 49-60, 1997.

[29] D. C. Henry Jr., R. B. Bettis, E. Riffer et al., "Comparison of oncedaily extended-release ciprofloxacin and conventional twicedaily ciprofloxacin for the treatment of uncomplicated urinary tract infection in women," Clinical Therapeutics, vol. 24, no. 12, pp. 2088-2104, 2002.

[30] A. Iravani, I. Klimberg, C. Briefer, C. Munera, S. F. Kowalsky, and R. M. Echols, "A trial comparing low-dose, short-course ciprofloxacin and standard 7 day therapy with co-trimoxazole or nitrofurantoin in the treatment of uncomplicated urinary tract infection," Journal of Antimicrobial Chemotherapy, vol. 43, supplement A, pp. 67-75, 1999.

[31] J. M. McCarty, G. Richard, W. Huck et al., "A randomized trial of short-course ciprofloxacin, ofloxacin, or trimethoprim/sulfamethoxazole for the treatment of acute urinary tract infection in women," American Journal of Medicine, vol. 106, no. 3, pp. 292-299, 1999.

[32] D. A. Talan, W. E. Stamm, T. M. Hooton et al., "Comparison of ciprofloxacin (7 days) and trimethoprim-sulfamethoxazole (14 days) for acute uncomplicated pyelonephritis in women: a randomized trial," JAMA, vol. 283, no. 12, pp. 1583-1590, 2000.

[33] C. A. M. McNulty, J. Richards, D. M. Livermore et al., "Clinical relevance of laboratory-reported antibiotic resistance in acute uncomplicated urinary tract infection in primary care," Journal of Antimicrobial Chemotherapy, vol. 58, no. 5, pp. 1000-1008, 2006.

[34] J. R. Johnson and W. E. Stamm, "Diagnosis and treatment of acute urinary tract infections," Infectious disease clinics of North America, vol. 1, no. 4, pp. 773-791, 1987.

[35] M. Rosenberg, "Pharmacoeconomics of treating uncomplicated urinary tract infections," International Journal of Antimicrobial Agents, vol. 11, no. 3-4, pp. 247-251, 1999.

[36] A. G. Carrie, C. J. Metge, D. M. Collins, G. K. M. Harding, and G. G. Zhanel, "Predictors of receipt of a fluoroquinolone versus trimethoprim-sulfamethoxazole for treatment of acute pyelonephritis in women in Manitoba, Canada," Pharmacoepidemiology and Drug Safety, vol. 13, no. 12, pp. 863-870, 2004.

[37] G. G. Zhanel, J. A. Karlowsky, G. K. M. Harding et al., "A Canadian national surveillance study of urinary tract isolates from outpatients: comparison of the activities of trimethoprimsulfamethoxazole, ampicillin, mecillinam, nitrofurantoin, and 
ciprofloxacin," Antimicrobial Agents and Chemotherapy, vol. 44, no. 4, pp. 1089-1092, 2000.

[38] N. Ceran, D. Mert, F. Y. Kocdogan et al., "A randomized comparative study of single-dose fosfomycin and 5-day ciprofloxacin in female patients with uncomplicated lower urinary tract infections," Journal of Infection and Chemotherapy, vol. 16, no. 6, pp. 424-430, 2010.

[39] K. Gupta, T. M. Hooton, and W. E. Stamm, "Isolation of fluoroquinolone-resistant rectal Escherichia coli after treatment of acute uncomplicated cystitis," Journal of Antimicrobial Chemotherapy, vol. 56, no. 1, pp. 243-246, 2005.

[40] M. A. Minassian, D. A. Lewis, D. Chattopadhyay, B. Bovill, G. J. Duckworth, and J. D. Williams, "A comparison between singledose fosfomycin trometamol (Monuril ${ }^{\circledR}$ ) and a 5-day course of trimethoprim in the treatment of uncomplicated lower urinary tract infection in women," International Journal of Antimicrobial Agents, vol. 10, no. 1, pp. 39-47, 1998.

[41] K. G. Naber and U. Thyroff-Friesinger, "Fosfomycin trometamol versus ofloxacin/Co-trimoxazole as single dose therapy of acute uncomplicated urinary tract infection in females: a multicentre study," Infection, vol. 18, supplement 2, pp. S70-S76, 1990.

[42] G. E. Stein, "Comparison of single-dose fosfomycin and a 7-day course of nitrofurantoin in female patients with uncomplicated urinary tract infection," Clinical Therapeutics, vol. 21, no. 11, pp. 1864-1872, 1999.

[43] E. Van Pienbroek, J. Hermans, A. A. Kaptein, and J. D. Mulder, "Fosfomycin trometamol in a single dose versus seven days nitrofurantoin in the treatment of acute uncomplicated urinary tract infections in women," Pharmacy World \& Science, vol. 15, no. 6, pp. 257-262, 1993.

[44] Ministry of Health and Long-Term Care, Ontario Drug Benefit Formulary/Comparative Drug Index, Ontario Ministry of Health and Long-Term Care, Toronto, Canada, 42nd edition, 2015.

[45] OCCI Ontario Case Costing Initiative, OCCI Costing Analysis Tool 2016, https://hsimi.on.ca/hdbportal/.

[46] Canadian Antimicrobial Resistance Alliance (CARA), 2011, http://www.can-r.com/index.php.

[47] J. A. Karlowsky, A. J. Denisuik, P. R. S. Lagacé-Wiens et al., "In vitro activity of fosfomycin against Escherichia coli isolated from patients with urinary tract infections in Canada as part of the CANWARD surveillance study," Antimicrobial Agents and Chemotherapy, vol. 58, no. 2, pp. 1252-1256, 2014.

[48] K. S. Ko, J. Y. Suh, K. R. Peck, M. Y. Lee, W. S. Oh, and K. T. Kwon, "In vitro activity of fosfomycin against ciprofloxacin-resistant or extended-spectrum beta-lactamase-producing Escherichia coli isolated from urine and blood," Diagnostic Microbiology and Infectious Disease, vol. 58, no. 1, pp. 111-115, 2007.

[49] Triton Pharma, Product Monograph: Monurol ${ }^{\circledR}$ (Fosfomycin Tromethamine) $3 g$ Sachet, Triton Pharma, Concord, Canada, 2012.

[50] G. Kahlmeter and P. Menday, "Cross-resistance and associated resistance in 2478 Escherichia coli isolates from the Pan-European ECO.SENS project surveying the antimicrobial susceptibility of pathogens from uncomplicated urinary tract infections," Journal of Antimicrobial Chemotherapy, vol. 52, no. 1, pp. 128-131, 2003.

[51] J. Reynaert, D. Van Eyck, and J. Vandepitte, "Single dose fosfomycin trometamol versus multiple dose norfloxacin over three days for uncomplicated UTI in general practice," Infection, vol. 18, no. 2, pp. S77-S79, 1990.
[52] J. W. Mouton, P. G. Ambrose, R. Canton et al., "Conserving antibiotics for the future: new ways to use old and new drugs from a pharmacokinetic and pharmacodynamic perspective," Drug Resistance Updates, vol. 14, no. 2, pp. 107-117, 2011.

[53] American College of Obstetricians and Gynecologists, "ACOG Practice Bulletin No. 91: treatment of urinary tract infections in nonpregnant women," Obstetrics \& Gynecology, vol. 111, no. 3, pp. 785-794, 2008.

[54] L. Almenar, B. Díaz, A. Quesada et al., "Cost-effectiveness analysis of cardiac resynchronization therapy in patients with NYHA i and nyha II heart failure in spain," International Journal of Technology Assessment in Health Care, vol. 29, no. 2, pp. 140146, 2013. 


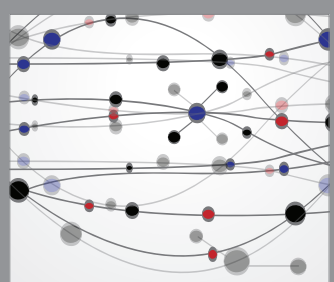

The Scientific World Journal
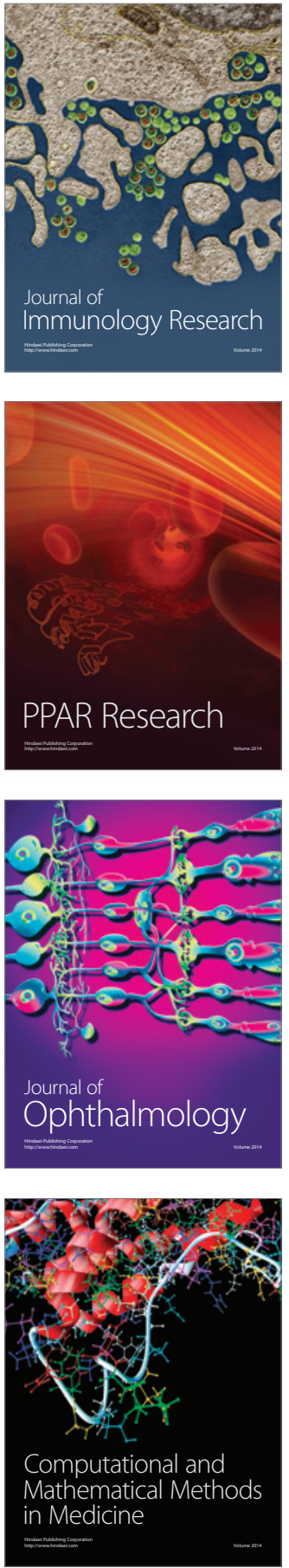

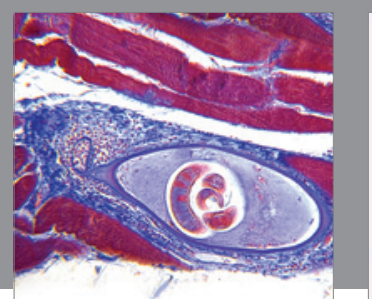

Gastroenterology Research and Practice
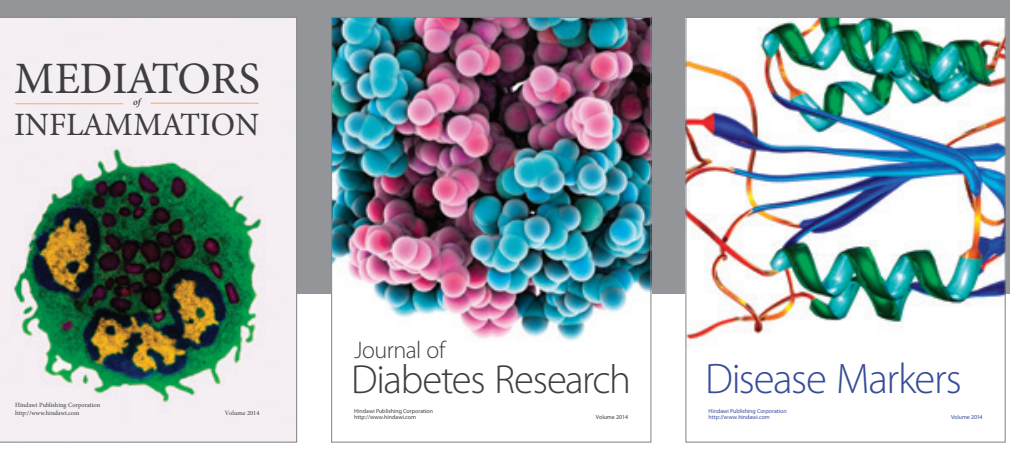

Disease Markers

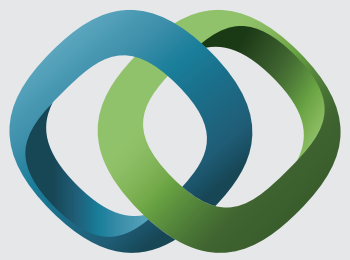

\section{Hindawi}

Submit your manuscripts at

https://www.hindawi.com
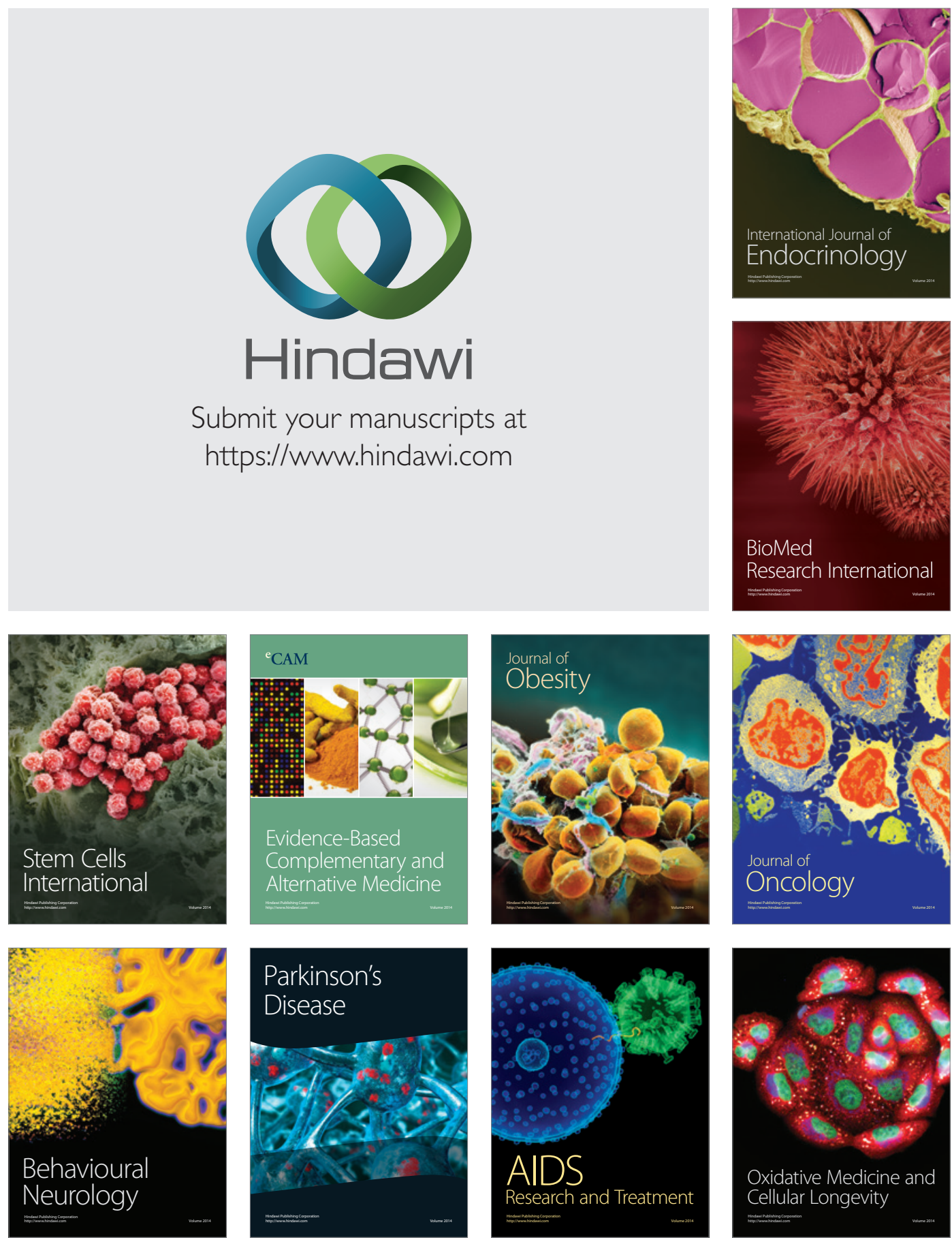